\title{
Long-Term Clinical Results of Double Bundle Reconstruction of the Medial Patellofemoral Ligament for Patellar Instability
}

\author{
Lei Zhang, MD ${ }^{1} \quad$ Zhiyao Li, MD ${ }^{1}$ \\ ${ }^{1}$ Department of Sports Medicine, Wangjing Hospital, \\ China Academy of Chinese Medical Sciences, Beijing, China \\ J Knee Surg 2019;32:153-159.
}

\begin{abstract}
Address for correspondence Zhiyao Li, MD, Department of Sports Medicine, Wangjing Hospital, China Academy of Chinese Medical Sciences, Beijing 100102, China (e-mail: li-zhi-yao@163.com).
\end{abstract}

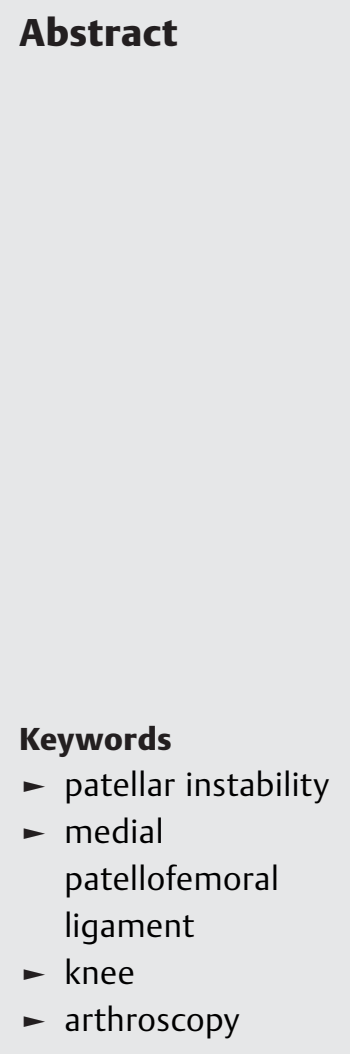

Medial patellofemoral ligament (MPFL) reconstruction is a satisfactory technique for patellar instability, and the anatomical double bundle variant is recommended for better clinical results. However, long-term outcomes are still uncharacterized. This study aimed to assess the effectiveness of double bundle reconstruction of the MPFL for patellar instability by means of established scores. A total of 68 patients with chronic patellar instability who underwent surgery from May 2005 to February 2010 were included prospectively. Anatomical double reconstruction of the MPFL with the semitendinosus tendon was conducted. Tegner Activity Scale (TAS), Kujala score, Lysholm knee score, and objective physical examination were assessed during followup. Median follow-up time for the patients was 8 (range, 6-10) years. Eight patients were lost to follow-up after 2 years. Preoperative mean TAS was $2.85 \pm 0.78$, increased to $4.91 \pm 0.84$ at 6 months and $7.26 \pm 0.78$ at 2 years postoperatively, and was $7.82 \pm 0.89$ at last follow-up. At last follow-up, pain free at rest was achieved in all patients; and 8 patients had knee pain in activities of daily living. Mean Kujala score was $57.53 \pm 8.59$ preoperatively, and increased to $61.22 \pm 6.46,89.51 \pm 3.90$, and $88.92 \pm 3.84$ at 6 months, 2 years postoperatively, and at last follow-up, respectively. Lysholm scores were also increased from preoperative values of $43.53 \pm 10.20$ to $58.22 \pm 6.80,89.37 \pm 4.38$, and $89.67 \pm 4.13$ at 6 months, 2 years postoperatively, and at last follow-up, respectively. Range of motion was $121.44 \pm 12.69$, $129.71 \pm 6.39,130.93 \pm 5.67$, and $130.78 \pm 5.80$ at preoperative point, 6 months, 2 years postoperatively, and at last follow-up, respectively. Long-term clinical results of double bundle reconstruction of the MPFL for patellar instability were encouraging.
The medial patellofemoral ligament (MPFL) is one of the most important stabilizers, preventing lateral dislocation of the patella. ${ }^{1-3}$ Recently, MPFL reconstruction has been advocated for the surgical treatment of patellar instability. Numerous techniques for MPFL reconstruction have been described, ${ }^{4-6}$ including nonanatomical or anatomical procedures. Anatomical double bundle reconstruction is an ideal way for the reconstruction of a ligament, because the MPFL is broader at

received

August 28, 2017

accepted after revision

January 28, 2018

published online

March 7, 2018 its patellar attachment than the femoral one. ${ }^{1,7}$ Several studies had described the short-term clinical results of the double bundle technique. ${ }^{8-11}$ However, long-term results are still uncharacterized. Results are needed for further evaluation of the effectiveness of the double bundle reconstruction. Therefore, this prospective cohort study aimed to clinically assess the long-term effectiveness of double bundle reconstruction of the MPFL for patellar instability.

Copyright $\odot 2019$ by Thieme Medical Publishers, Inc., 333 Seventh Avenue, New York, NY 10001, USA.

DOI https://doi.org/ 10.1055/s-0038-1636913. ISSN 1538-8506. 


\section{Materials and Methods}

\section{Inclusion and Exclusion Criteria}

The inclusion criteria were: (1) chronic patellar instability, described as two or more patellar dislocations, with a persistent apprehension sign after a rehabilitation protocol applied for 6 months; (2) closed growth plate; (3) $<20^{\circ}$ of $\mathrm{Q}$ angle and $<1.3$ of patellar height by the method of Insall and Salvati; ${ }^{12}$ and (4) tibial external rotation angle of $<8^{\circ}$. Exclusion criteria were: (1) arthrosis or generalized ligamentous laxity; (2) concurrent ligament (e.g., anterior cruciate ligament $[\mathrm{ACL}]$ and posterior cruciate ligament [PCL]) or meniscus injury; (3) grades 3 to 4 chondral damage; (4) significant trochlear dysplasia (Dejour type B, C, or D) ${ }^{13}$ (5) history of knee surgery; and (6) fracture around the knee.

\section{Patients}

In this prospective study, 68 patients with patellar instability were evaluated before and after surgical treatment, which was performed from May 2005 to February 2010. They included 22 male and 46 female patients with a mean age of 21 years (16-32) at first admission. Median duration of preoperative patellar instability was 12 months (range from 6 months to 3 years).

\section{Surgical Methods}

The surgery was performed as previously described, ${ }^{8,14}$ with minor modifications. Briefly, the patient in the supine position was submitted to spinal anesthesia, and was confirmed of patellar instability by knee arthroscopy. Then, orientation and tracking of the patella were recorded from the anteromedial portal site. The semitendinosus tendon was freed through a 2- to 3-cm longitudinal incision near the tibial insertion of the pes anserinus tendon. A 4- to 5-cm incision was made longitudinally $2 \mathrm{~cm}$ off the medial aspect of the patella. A bony rim was created on the medial side of the patella, where the MPFL attachment was located. Two suture anchors (GIITM, DePuy Mitek, Raynham, MA) equipped with no. 3 nonabsorbable braided suture were placed into the middle point and superomedial pole of the patella. Afterward, the adductor tubercle was identified at the medial condyle of the femur. The natural MPFL attachment is distal to the tubercle. When proper Kirschner wire (K-wire) placement was achieved with an isometric test using braided suture, from anchors to K-wire, a 7-mm diameter tunnel was drilled over the guidewire. The middle part of the graft was sutured to the anchor at the patella. The graft ends were pulled into the bone tunnel by removing the guidewire through the femoral condyle. Tensioning of the graft was performed according to a realigned patellar tracking throughout the knee's range of motion (ROM). After the patella was manually positioned at the proper location in the femoral groove, the tendon graft was fixed by an absorbable soft-tissue interference screw (BIOCRYL Interference Screw, DePuy Mitek) at $30^{\circ}$ of knee flexion (-Fig. 1). Arthroscopy was performed anew to assess patella position in the trochlea, if they were perfectly matched after MPFL reconstruction.

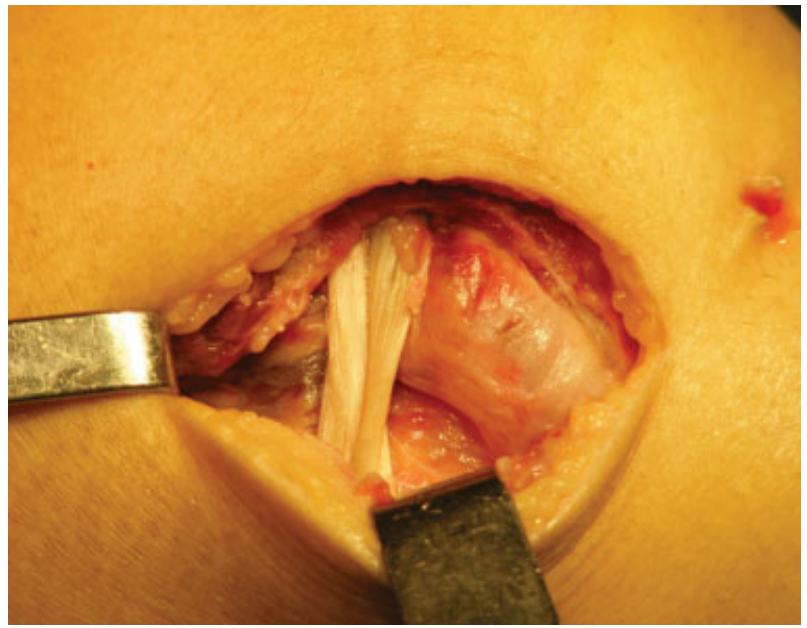

Fig. 1 Medial patellofemoral ligament (MPFL) reconstruction. After manual positioning of the patella in the femoral groove, the tendon graft was fixed by an absorbable soft-tissue interference screw at $30^{\circ}$ of knee flexion. A perioperative image depicting the reconstructed double bundle MPFL is shown.

\section{Postoperative Rehabilitation}

Immediately after surgery, the knee was placed in a simple leg brace. Straight leg-raising and quadriceps setting exercises were started as soon as tolerated by the patient. The patients walked on a full-extended knee in the first 2 weeks with full weight-bearing. From 2 to 4 weeks, patients exercised with an allowed ROM of 0 to $90^{\circ}$ and walked with full weight-bearing. From 4 to 6 weeks, patients exercised with a ROM reaching $120^{\circ}$, and walked with full weight-bearing. After 6 weeks, patients could get full ROM, and free activity was allowed. Individual (e.g., running) and contact (e.g., basketball) sports were allowed after 3 and 6 months, respectively.

\section{Follow-Up and Outcomes Assessment}

All patients were assessed at four time points: preoperatively; 6 months and 2 years postoperatively; and at last follow-up (in June 2016). Eight patients were lost after 2 years; there were 60 patients in the last follow-up. Median follow-up was 8 years, ranging from 6 to 10 years.

Subjective patient evaluation included activity level and pain, and was performed using the Tegner Activity Scale (TAS), a standardized method for grading work and sporting activities. ${ }^{15}$ Kujala scoring was also applied to all patients at each time point. The Kujala score is special for patellofemoral joint assessment, and ranges from 0 to 100 , with 100 representing the best value. The Lysholm score, ${ }^{16}$ the most commonly used for knee joint evaluation, contains 8 subscores ranging from 0 to 100 , with 100 as the best value. The objective physical examination included palpable pain, apprehension signs, and ROM. Complications as delayed union of the incision, skin hypoesthesia, and patellar clicking at knee flexion were considered. Postoperative dislocation was requested immediately, and treatment was decided by an orthopedist. This information was also recorded during follow-up. 


\section{Statistical Analysis}

Statistical analyses were performed with the SPSS software version 15.0 (SPSS, Chicago, IL). For comparison, the paired sample $t$-test and independent sample $t$-test was used. $p$ Value of $<0.05$ was considered statistically significant.

\section{Results}

\section{Tegner Activity Scale}

Activity level of the patients was assessed by the TAS. Preoperative mean TAS was $2.85 \pm 0.78$, increased to $4.91 \pm 0.84$ at 6 months and $7.26 \pm 0.78$ at 2 years postoperatively, and was $7.82 \pm 0.89$ at last follow-up. At last follow-up, all patients were completely pain free at rest (-Table 1); 52 patients were pain-free when performing activities of daily living (Tegner level 4) (-Table 1). Meanwhile, 14 patients were pain-free when performing sporting activities (Tegner level 7) (-Table 1). At last follow-up, 45 patients felt that their knee function had improved in terms of activities of daily living (Tegner level 4) comparing to the preoperative point; meanwhile, 46 patients indicated that their ability to perform recreational sports activities had improved (Tegner level 7) comparing to the preoperative point; 18 patients could do regular contact sports (Tegner level 8).

\section{Kujala Score}

Preoperative Kujala knee function scores were $57.53 \pm 8.59$ points (range, 36-79 points), and increased to $61.22 \pm 6.46$ (range, $42-75$ points) at 6 months after operation, $89.51 \pm 3.90$ (range, 75-95 points) and $88.92 \pm 3.84$ (range, 73-95 points), respectively, at 2 years postoperatively and last follow-up. Detailed information is provided in - Table 2 for all time points.

\section{Lysholm Score}

The preoperative Lysholm scores is $43.53 \pm 10.20$ points (range, 23-65 points), and it increased to $58.22 \pm 6.80$ (range, 42-75 points) at 6 months after operation, $89.37 \pm 4.38$ (range, $78-96$ points) and $89.67 \pm 4.13$ (range, 80-96 points), respectively, at 2 years postoperatively and last follow-up. Detailed information is provided in - Table 3 for all time points.

\section{Physical Examination Results}

Physical examination included palpable pain, apprehension signs, and ROM. At the last follow-up, palpable pain was found in $8.33 \%(5 / 60)$ and $13.33 \%(8 / 60)$ patients at the medial patellar edge and medial femoral condyle, respectively. There were no positive apprehension signs or passive patellar hypermobility. ROM was $121.44 \pm 12.69$, $129.71 \pm 6.39,130.93 \pm 5.67$, and $130.78 \pm 5.80$ at preoperative point, 6 months, 2 years postoperatively, and at last follow-up, respectively. Fifty-nine patients had a normal ROM of the knee. One patient had loss of hyperextension of the knee, with flexion reduced by $10^{\circ}$ compared with the contralateral knee. The detailed data regarding these changes are summarized in -Table 4 .

\section{Complications}

There was no intraoperative complication, such as fracture or failure of fixation. The fixation by anchors allowed little bone loss of patella, which allowed avoidance of patella fracture in this series. A major complication was delayed union of the incision in two obese patients, resulting from liquefaction of the subcutaneous fat tissue. They had a secondary closure of the incision. Meanwhile, three patients had skin hypoesthesia on the anterior knee, maybe due to injury of the infrapatellar branch of the saphenous nerve. The area was $\sim 6 \mathrm{~cm}^{2}$ in average. Two patients had patellar clicking at knee flexion of 30; however, it was mild with no interference with daily life activities. No further treatments were applied to these patients. No redislocation was observed, and there was no revision of the reconstruction.

\section{Discussion}

To get good long-term clinical results, patient selection and procedure decision is critical. Using combined procedures with MPFL reconstruction is controversial. MPFL reconstruction alone is effective in treating patellar instability, ${ }^{9}$ but strict patient selection is very critical for good long-term clinical results. This study included patients with patellar instability and normal alignment of the lower limb, who did not need combined procedures. In our opinion, additional procedures, such as medial tibial tuberosity transfer combined with MPFL reconstruction, are necessary in cases of

Table 1 Pain as evaluated by the patients at various time points according to Tegner activity level

\begin{tabular}{|l|l|l|l|l|l|l|l|}
\hline \multirow{2}{*}{} & \multicolumn{2}{|l|}{ Pain at rest } & \multicolumn{2}{l|}{$\begin{array}{l}\text { Pain during activities } \\
\text { of daily living } \\
\text { Tegner level 4 }\end{array}$} & \multicolumn{2}{l|}{$\begin{array}{l}\text { Pain during sports } \\
\text { activities } \\
\text { Tegner level 7 }\end{array}$} & Tegner Activity Scale \\
\cline { 2 - 8 } & Positive & Negative & Positive & Negative & Positive & Negative & \\
\hline Preop $(n=68)$ & 13 & 55 & 45 & 23 & 68 & 0 & $2.85 \pm 0.78$ \\
\hline 6 mo $(n=68)$ & 10 & 58 & 25 & 43 & 30 & 38 & $4.91 \pm 0.84$ \\
\hline 2 y $(n=68)$ & 0 & 68 & 7 & 61 & 15 & 53 & $7.26 \pm 0.78$ \\
\hline Last follow-up $(n=60)$ & 0 & 60 & 8 & 52 & 46 & 14 & $7.82 \pm 0.89$ \\
\hline
\end{tabular}

Note: Data note of Tegner Activity Scale (a/ Preop, b/ 6 mo, c/ 2 y, d/ Last follow-up). Data comparison: a/b $(t=-15.965, p=0.000)$, a/c $(t=-34.053, p=0.000), \mathrm{a} / \mathrm{d}(t=-33.627, p=0.000), \mathrm{b} / \mathrm{c}(t=-15.762, p=0.000), \mathrm{b} / \mathrm{d}(t=-18.942, p=0.000), \mathrm{c} / \mathrm{d}(t=-3.724, p=0.000)$. 

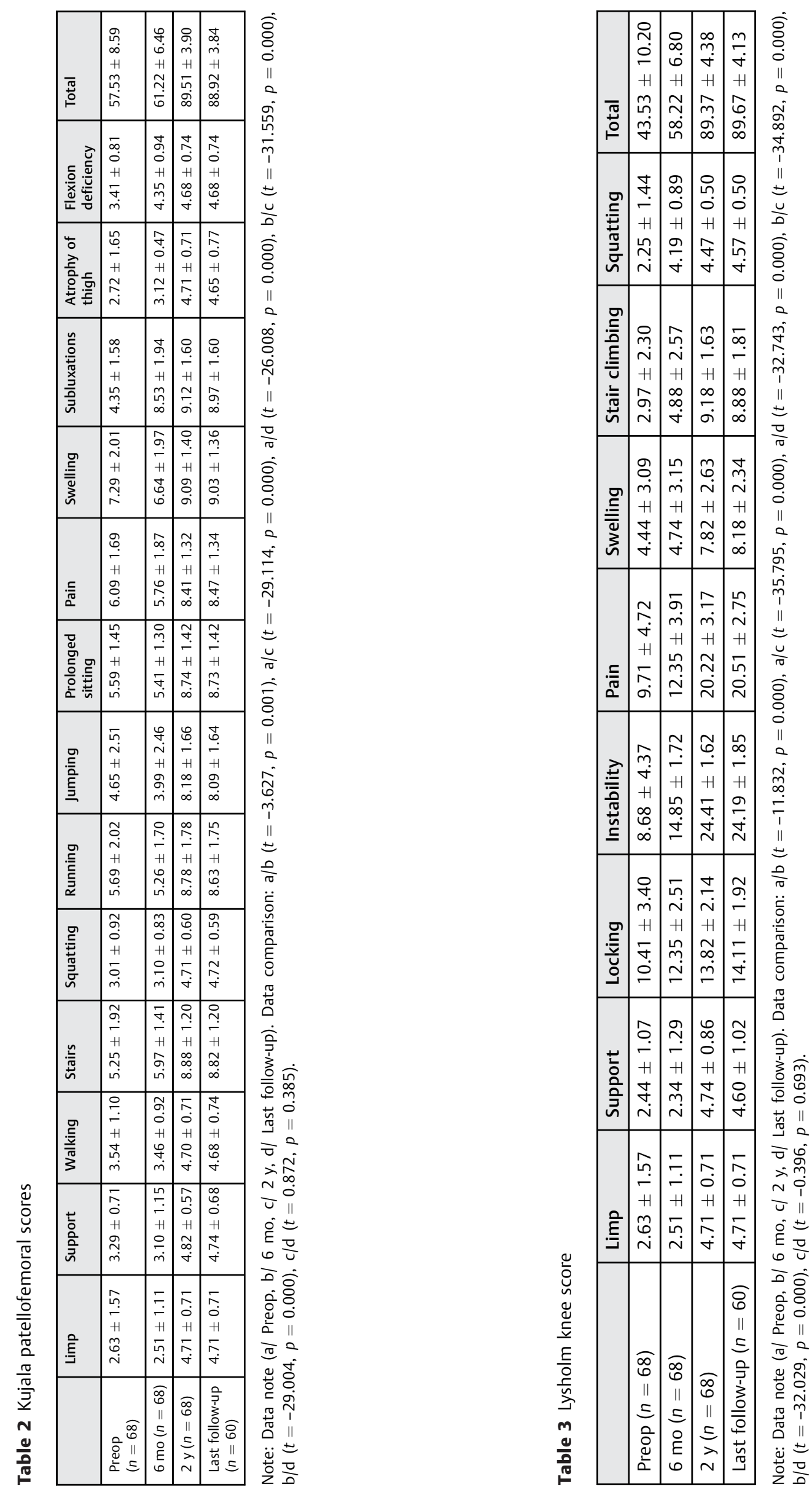
Table 4 Changes of physical examination

\begin{tabular}{|l|l|l|l|l|l|l|l|}
\hline \multirow{2}{*}{} & \multicolumn{2}{|l|}{$\begin{array}{l}\text { Palpable pain on } \\
\text { medial patella }\end{array}$} & \multicolumn{2}{l|}{$\begin{array}{l}\text { Palpable pain on } \\
\text { medial epicondyle }\end{array}$} & \multicolumn{2}{l}{ Apprehension test } & \multirow{2}{*}{ Range of motion } \\
\cline { 2 - 7 } & Positive & negative & Positive & negative & Positive & negative & \\
\hline Preop $(n=68)$ & 45 & 23 & 10 & 58 & 53 & 15 & $121.44 \pm 12.69$ \\
\hline 6 mo $(n=68)$ & 33 & 35 & 56 & 12 & 17 & 51 & $129.71 \pm 6.39$ \\
\hline 2 y $(n=68)$ & 7 & 61 & 12 & 56 & 3 & 65 & $130.93 \pm 5.67$ \\
\hline Last follow-up $(n=60)$ & 5 & 55 & 8 & 52 & 0 & 60 & $130.78 \pm 5.80$ \\
\hline
\end{tabular}

Note: Data note of range of motion (a/ Preop, b/ 6 mo, c/ 2 y, d/ Last follow-up). Data comparison: a/b $(t=-5.102, p=0.000), a / c(t=-5.527$, $p=0.000), \mathrm{a} / \mathrm{d}(t=-5.205, p=0.000), \mathrm{b} / \mathrm{c}(t=-2.735, p=0.008), \mathrm{b} / \mathrm{d}(t=-0.977, p=0.330), \mathrm{c} / \mathrm{d}(t=0.138, p=0.890)$.

large $\mathrm{Q}$ angles. Therefore, patients who had grossly abnormal $\mathrm{Q}$ angles (lager than $20^{\circ}$ ) or significant trochlear dysplasia (Dejour type B, C, or D) were excluded from this study. There is also a controversy about the function of lateral release in the treatment of patellar instability. In a normal knee, lateral displacement of the patella causes its lateral edge to become more prominent. This maneuver tightens the lateral retinaculum: a tight band can be palpated. Christoforakis et $\mathrm{al}^{17}$ found that the patella can be displaced laterally with significantly less force when the knee is near extension. Thus, in the normal knee, the lateral retinaculum is a restraint to patellar lateral displacement. In our opinion, lateral release should be performed only in patients with severe tightness of lateral patellar structures which is called excessive lateral pressure syndrome. The good clinical outcome of this study demonstrated that lateral release is not necessary for patellar instability without excessive lateral pressure syndrome.

To get good long-term clinical results, anatomical double bundle technique may be optimal for MPFL reconstruction. First, ligamentous attachments are critical for anatomical procedures, anatomical insertion is important. Nonanatomical and anatomical techniques are well described previously. ${ }^{4-7,18-20}$ Nonanatomical surgical techniques alter the premorbid patella mechanics by several principles. Biomechanical studies have demonstrated that the length change pattern of a MPFL reconstruction depends critically on the attachment site. ${ }^{21}$ However, a controversy remains regarding the exact patellar insertion of the MPFL. Schöttle et $\mathrm{al}^{9}$ inserted it at "the proximal one-third aspect of the patella," while Tang et a ${ }^{10}$ used "the superomedial border of the patella" as insertion site; Christiansen et al ${ }^{11}$ placed the two bundles "in the proximal two-thirds of the patella, 10 to $15 \mathrm{~mm}$ apart." Recently, Schöttle et $\mathrm{al}^{8}$ inserted it at "the proximal and distal ends of the medial edge," while Siebold et $\mathrm{al}^{22}$ placed the two bundles "in the superior-medial corner and in the center of the medial patella rim." The method by Siebold et $\mathrm{al}^{22}$ seems more reasonable according to an anatomical study by Amis et al. ${ }^{1}$ In this study, the two bundles were placed in the superior-medial corner and the center of the medial patella rim according to an anatomical study by Amis et al, ${ }^{1}$ rather than the proximal and distal ends of the medial aspect. ${ }^{8}$ Second, ligament shape is critical for anatomical procedures too. The MPFL is broader at its patellar attachment than the femoral one, with a sail-like shape with proximal and distal bundles. ${ }^{1,23}$ Single point fixation at the patellar side can increase the rotational moment of the patella in flexion extension movement. The double bundle MPFL better restores the anatomy, avoiding patella rotation. ${ }^{24,25}$ In this study, a laminar attachment is performed on the patellar side, creating a proximal and a distal bundle. This seems to provide a higher stability during flexion, decreasing patellar rotation, unlike the techniques where only a single-point fixation is performed. ${ }^{26-29}$ In the most recent research, the medial quadriceps tendon-femoral

Table 5 Clinical results of double bundle MPFL reconstruction of previous studies and this study

\begin{tabular}{|c|c|c|c|c|c|c|}
\hline & Author & No. of cases & Follow-up time & $\begin{array}{l}\text { Kujala } \\
\text { preop and postop }\end{array}$ & $\begin{array}{l}\text { Lysholm } \\
\text { preop and postop }\end{array}$ & $\begin{array}{l}\text { Tegner } \\
\text { preop and postop }\end{array}$ \\
\hline 1 & Sadigursky et $\mathrm{al}^{33}$ & 31 & $12 \mathrm{mo}$ & $45.6-94.0$ & $40.5-91.6$ & $3-4.4$ \\
\hline 2 & Gonçaives et $\mathrm{al}^{34}$ & 24 & $26 \mathrm{mo}$ & $59.8-83.5$ & $53.7-93.3$ & No data \\
\hline 3 & Kodkani ${ }^{35}$ & 56 & $26 \mathrm{mo}$ & $64.3-99.7$ & No data & No data \\
\hline 4 & Vavalle and Capozzi ${ }^{36}$ & 16 & $38 \mathrm{mo}$ & $35.8-88.8$ & $43.3-89.3$ & No data \\
\hline 5 & Wang et al ${ }^{37}$ & 26 & $38 \mathrm{mo}$ & $53.2-89.4$ & $59.6-90.3$ & $3.1-6.2$ \\
\hline 6 & Wang et $\mathrm{al}^{38}$ & 44 & $48 \mathrm{mo}$ & $61.0-92.9$ & No data & No data \\
\hline 7 & Li et $\mathrm{al}^{39}$ & 65 & $78 \mathrm{mo}$ & $52.9-90.1$ & $47.2-92.5$ & $3.1-5.8$ \\
\hline 8 & Zhang and Li (this study) & 60 & $96 \mathrm{mo}$ & $57.5-88.9$ & $43.5-89.7$ & $3.0-5.4$ \\
\hline
\end{tabular}

Abbreviation: MPFL, medial patellofemoral ligament. 
ligament (MQTFL) was reported as an important part of medial stabilizer. $2,7,30,31$ Combined reconstruction of the MPFL and MQTFL had received good clinical results for patellar instability in children and adolescents. ${ }^{32}$ But in this study, the author did not attach great importance to MQTFL several years ago. It will be an important consideration in future research.

In this study, we found that anatomical double bundle reconstruction of MPFL alone can give a good function of medial stability, and this technique can get good short-term and long-term clinical results. The clinical scores increased rapidly from 6 months to 2 years after the operation, and then keep that level to 10 years, without attenuation. The long-term clinical results were encouraging.

We compare the clinical results to other studies with the same technique ${ }^{33-39}$ (-Table 5). All of the studies used the Kujala score, then most of them used the Lysholm score, some of them used the Tegner score. The Kujala score was around 90 at 24 months after operation, similar to our results. During the follow-up time increase, the Kujala score did not change significantly. It was similar to our study too, and the Kujala score can stay around 90 up to 8 years.

A limitation should be mentioned of this study. Follow-up radiographic or arthroscopic data were not available. However, patients typically returned to highest level of activity possible at last follow-up and further imaging or arthroscopy may not be necessary for these patients.

\section{Conclusion}

In conclusion, the long-term results of anatomical double bundle reconstruction of the MPFL for chronic patellar instability are effective.

\section{Conflict of Interest}

None.

\section{References}

1 Amis AA, Firer P, Mountney J, Senavongse W, Thomas NP. Anatomy and biomechanics of the medial patellofemoral ligament. Knee 2003;10(03):215-220

2 Tanaka MJ. The anatomy of the medial patellofemoral complex. Sports Med Arthrosc Rev 2017;25(02):e8-e11

3 Trinh TQ Ferrel JR, Bentley JC, Steensen RN. The anatomy of the medial patellofemoral ligament. Orthopedics 2017;40(04):e583-e588

4 Dragoo JL, Nguyen M, Gatewood CT, Taunton JD, Young S. Medial patellofemoral ligament repair versus reconstruction for recurrent patellar instability: two-year results of an algorithm-based approach. Orthop J Sports Med 2017;5(03):2325967116689465

5 Fink C, Veselko M, Herbort M, Hoser C. MPFL reconstruction using a quadriceps tendon graft: part 2: operative technique and short term clinical results. Knee 2014;21(06):1175-1179

6 Andrish J. Surgical options for patellar stabilization in the skeletally immature patient. Sports Med Arthrosc Rev 2017;25(02): 100-104

7 Tanaka MJ, Voss A, Fulkerson JP. The anatomic midpoint of the attachment of the medial patellofemoral complex. J Bone Joint Surg Am 2016;98(14):1199-1205

8 Schöttle PB, Hensler D, Imhoff AB. Anatomical double-bundle MPFL reconstruction with an aperture fixation. Knee Surg Sports Traumatol Arthrosc 2010;18(02):147-151
9 Schöttle PB, Fucentese SF, Romero J. Clinical and radiological outcome of medial patellofemoral ligament reconstruction with a semitendinosus autograft for patella instability. Knee Surg Sports Traumatol Arthrosc 2005;13(07):516-521

10 Tang $\mathrm{H}, \mathrm{Xu} \mathrm{YQ}$, Zheng TE, et al. Anatomical double bundle reconstruction of medial patellofemoral ligament with allograft tendon in the treatment of patellar dislocations. Zhongguo $\mathrm{Gu}$ Shang 2015;28(03):252-255

11 Christiansen SE, Jacobsen BW, Lund B, Lind M. Reconstruction of the medial patellofemoral ligament with gracilis tendon autograft in transverse patellar drill holes. Arthroscopy 2008;24(01):82-87

12 Grelsamer RP, Meadows S. The modified Insall-Salvati ratio for assessment of patellar height. Clin Orthop Relat Res 1992;(282): 170-176

13 Botchu R, Obaid H, Rennie WJ. Correlation between trochlear dysplasia and the notch index. J Orthop Surg (Hong Kong) 2013;21 (03):290-293

14 Singhal R, Rogers S, Charalambous CP. Double-bundle medial patellofemoral ligament reconstruction with hamstring tendon autograft and mediolateral patellar tunnel fixation: a metaanalysis of outcomes and complications. Bone Joint J 2013;95-B (07):900-905

15 Gobbi A, Whyte GP. One-stage cartilage repair using a hyaluronic acid-based scaffold with activated bone marrow-derived mesenchymal stem cells compared with microfracture: fiveyear follow-up. Am J Sports Med 2016;44(11):2846-2854

16 Tegner Y, Lysholm J. Rating systems in the evaluation of knee ligament injuries. Clin Orthop Relat Res 1985;(198):43-49

17 Christoforakis J, Bull AM, Strachan RK, Shymkiw R, Senavongse W, Amis AA. Effects of lateral retinacular release on the lateral stability of the patella. Knee Surg Sports Traumatol Arthrosc 2006;14(03):273-277

18 Longo UG, Berton A, Salvatore G, et al. Medial patellofemoral ligament reconstruction combined with bony procedures for patellar instability: current indications, outcomes, and complications. Arthroscopy 2016;32(07):1421-1427

19 Ambrožič B, Novak S. The influence of medial patellofemoral ligament reconstruction on clinical results and sports activity level. Phys Sportsmed 2016;44(02):133-140

20 Houdek CG, Esquivel AO, Cracchiolo AM, Lemos SE. A biomechanical comparison of isometric and anatomic medial patellofemoral ligament reconstruction. J Knee Surg 2016;29(06): 522-526

21 Russo F, Doan J, Chase DC, Farnsworth CL, Pennock AT. Medial patellofemoral ligament reconstruction: fixation technique biomechanics. J Knee Surg 2016;29(04):303-309

22 Siebold R, Chikale S, Sartory N, Hariri N, Feil S, Pässler HH. Hamstring graft fixation in MPFL reconstruction at the patella using a transosseous suture technique. Knee Surg Sports Traumatol Arthrosc 2010;18(11):1542-1544

23 Kang HJ, Wang F, Chen BC, Su YL, Zhang ZC, Yan CB. Functional bundles of the medial patellofemoral ligament. Knee Surg Sports Traumatol Arthrosc 2010;18(11):1511-1516

24 Wang Q, Huang W, Cai D, Huang H. Biomechanical comparison of single- and double-bundle medial patellofemoral ligament reconstruction. J Orthop Surg 2017;12(01):29

25 Kita K, Tanaka Y, Toritsuka Y, et al. Factors affecting the outcomes of double-bundle medial patellofemoral ligament reconstruction for recurrent patellar dislocations evaluated by multivariate analysis. Am J Sports Med 2015;43(12):2988-2996

26 Pemmaraju G, Bassett J, Abbas R, Nagra G, Chugh S, Mughal E. Outcomes of combined tibial tuberosity transfer and medial patellofemoral ligament reconstruction for recurrent patellar instability. Acta Orthop Belg 2016;82(02):365-371

27 Suganuma J, Mochizuki R, Shibata R, et al. Reconstruction of the medial patellofemoral ligament with arthroscopic control of patellofemoral congruence using electrical stimulation of the quadriceps. Arthrosc Tech 2016;5(03):e649-e656 
28 Valkering KP, Rajeev A, Caplan N, Tuinebreijer WE, Kader DF. An evaluation of the effectiveness of medial patellofemoral ligament reconstruction using an anatomical tunnel site. Knee Surg Sports Traumatol Arthrosc 2017;25(10):3206-3212

29 Gobbi RG, Pereira CA, Sadigursky D, et al. Evaluation of the isometry of different points of the patella and femur for medial patellofemoral ligament reconstruction. Clin Biomech (Bristol, Avon) 2016;38:8-12

30 Fulkerson JP, Edgar C. Medial quadriceps tendon-femoral ligament: surgical anatomy and reconstruction technique to prevent patella instability. Arthrosc Tech 2013;2(02):e125-e128

31 Mochizuki T, Nimura A, Tateishi T, Yamaguchi K, Muneta T, Akita K. Anatomic study of the attachment of the medial patellofemoral ligament and its characteristic relationships to the vastus intermedius. Knee Surg Sports Traumatol Arthrosc 2013;21(02): 305-310

32 Redler LH, Spang RC, Tepolt F, Davis EA, Kocher MS. Combined reconstruction of the medial patellofemoral ligament (MPFL) and medial quadriceps tendon-femoral ligament (MQTFL) for patellar instability in children and adolescents: surgical technique and outcomes. Orthop J Sports Med 2017;5(7, Suppl 6). Doi: 10.1177/ 2325967117500387

33 Sadigursky D, de Melo Laranjeira MS, Nunes M, Caneiro RJ, Colavolpe PO. Reconstruction of the medial patellofemoral liga- ment by means of the anatomical double-bundle technique using metal anchors. Rev Bras Ortop 2016;51(03):290-297

34 Gonçaives MB, Júnior LH, Soares LF, Gonçaives TJ, Dos Santos RL, Pereira ML. Medial patellofemoral ligament reconstruction to treat recurrent patellar dislocation. Rev Bras Ortop 2015;46 (02):160-164

35 Kodkani PS. "Basket weave technique" for medial patellofemoral ligament reconstruction: clinical outcome of a prospective study. Indian J Orthop 2016;50(01):34-42

36 Vavalle G, Capozzi M. Isolated reconstruction of the medial patellofemoral ligament with autologous quadriceps tendon. J Orthop Traumatol 2016;17(02):155-162

37 Wang HD, Dong JT, Gao SJ. Medial patellofemoral ligament reconstruction using a bone groove and a suture anchor at patellar: a safe and firm fixation technique and 3-year followup study. J Orthop Surg 2016;11(01):138

38 Wang CH, Ma LF, Zhou JW, et al. Double-bundle anatomical versus single-bundle isometric medial patellofemoral ligament reconstruction for patellar dislocation. Int Orthop 2013;37(04): 617-624

39 Li J, Li Y, Wei J, Wang J, Gao S, Shen Y. A simple technique for reconstruction of medial patellofemoral ligament with bonefascia tunnel fixation at the medial margin of the patella: a 6year-minimum follow-up study. J Orthop Surg 2014;9:66 